\title{
Pharmakologische Beeinflussung der Ansammlung und Resorption des Exsudates bei experimenteller Pleuritis.
}

I. Mitteilung: Pyrazolonderivate und Calcium.

Von

Takanori Kurota.

(黑田高德)

(Aus der Medizinischen Klinik von Prof. Dr. T.

$\mathrm{K}$ a to, Tohoku Reichsuniversität

zu Sendai.)

(Received for publication, March 9, 1945)

Die Pleuritis ist eine der Krankheiten, welche klinisch sehr häufig beobachtet werden. Darum liegen über sie zahlreiche klinisch-experimentelle Angaben vor. Diese sind jedoch meist alt und ausserdem verhältnismässig selten Arbeiten, die vom Standpunkt der modernen pathologischen Physiologie aus gemacht wurden. Besonders gering ist die Anzahl derer, welche die therapeutische Seite der Ansammlung und Resorption des Exsudates behandeln. Unser Kollege $\mathrm{H}$ a y a si' il $^{\prime}$ reichte Pleuritikern Pyrazolonderivate dar und bestimmte die Änderungen des Eiweisses und des kolloid-osmotischen Druckes in Exsudat und Blut. Auf Grund seiner Versuchsergebnisse behauptete er, dass die von ihm dargereichten Medikamente die Resorption des Exsudates bei Pleuritikern begünstigen. Wenn man aber Versuche bei Menschenpleuritis durchführt, so ist es manchmal wegen verschiedener Hemmnisse schwierig, vom wirklichen Anbeginn der Krankheit an Beobachtungen zu machen und Blut und Exsudat regel und planmässig zu untersuchen.

Indem wir daher bei Kaninchen durch Einführung von Ameisensäure in die Pleurahöhle experimentell eine entzündliche Exsudatansammlung herbeiführten, haben wir vorliegende Versuche durchgeführt um den Einfluss verschiedener Medikamente auf die Ansammlung und Resorption des Exsudates zu untersuchen.

Obgleich man selbstverständlich diese Ergebnisse nicht sofort zur Therapie der Pleuritis bei Menschen anwenden kann, so können sie doch wenigstens bei der Behandlung mitberücksichtigt werden. Vielleicht kann man sie nicht nur bei Pleuritis, sondern auch bei allerlei 
anderen serösen Entzündungen des menschlichen Körpers anwenden. Dass die Ansammlung und Resorption des entzündlichen Exsudates nebst den Änderungen der Permeabilität der serösen Membran mit der physikalischen wasseranziehenden Kraft des Blutes und Exsudates, insbesondere mit dem kolloid-osmotischen Druck (im folgenden kurz mit k.o.D. bezeichnet) der Eiweisskörper in Blut und Exsudat in irgendeiner Beziehung stehen, ist schon von einer Reihe von Forschern (I versen u. Nakazawa, ${ }^{2)}$ Kylin, ${ }^{3)}$ Meyer u. Friedheim, ${ }^{4}$ Iversen u. Johansen, ${ }^{5}$ ' Nishiyama, ${ }^{6}$ Hayasi $i^{11}$ ) angedeutet worden. Darum wurden in unseren Versuchen ebenfalls Eiweiss und sein k.o.D. im Exsudat dem Verlaufe nachfolgend bestimmt und mit den entsprechenden Werten des Blutes verglichen, um festzustellen, ob irgendwelche Beziehungen zwischen ihnen bestehen.

\section{Versuchsmethode.}

Als Versuchstiere dienten gesunde erwachsene Kaninchen von ungefähr $2 \mathrm{~kg}$ Körpergewicht. In die Pleurahöhle der einen Seite wurde 1

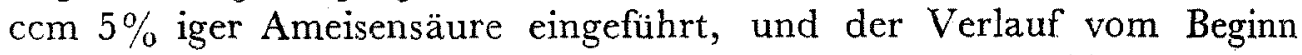
der durch ihren Reiz verursachten Exsudatansammlung bis zu seiner Resorption beobachtet. Der Beginn der Exsudation und ihr Verschwinden wurden mit Hilfe von Röntgenaufnahmen oder Probepunktionen festgestellt. Proben von je $1,5 \mathrm{ccm}$ Blut und Exsudat wurden morgens vor der Futterverabreichung entnommen. Vor Beginn des eigentlichen Versuches wurde eine Blutprobe gemacht, und wenn nach Einführung der Ameisensäure die Exsudation begonnen hatte, wurden täglich oder alle zwei Tage fast zur gleichen Zeit Probepunktionen des Exsudates und Blutproben durchgeführt. Der Eiweissgehalt der entnommenen Proben wurden mittelst $\mathrm{Pulf}$ i ich'schen Eintauchrefraktmeters und der k.o.D. nach der Methode von $\mathrm{Krogh}$ und $\mathrm{Nakazawa}$ ) bestimmt. Die Anwendungsweise der gebrauchten Medikamente ist an den betreffenden Stellen angegeben.

\section{Kontrollbeobachtungen.}

Zur Kontrolle wurde bei 7 Kaninchen ausser Ameisensäure kein anderes Mittel verabreicht, die durch sie hervorgerufene Ansammlung des Exsudates und seine Resorption beobachtet, und die Eiweisskörper und der k.o.D. in Blut und Exsudat dem Verlauf nachfolgend bestimmt. Aus den Resultaten ergab sich, dass die Exsudation nach Ablauf von 16-18 Stunden nach der Ameisensäureeinführung beginnt und, wie röntgenologisch nachgewiesen werden konnte, nach 4-5 Tagen die ganze betreffende Pleurahöhle mit Exsudat angefüllt ist. Danach nimmt das Exsudat allmählich ab, und bẹi 4 Fällen von 7 Versuchstieren ist es 
am 12 Tage, bei den 3 übrigen jeweils am 17, 18 und 19 Tage vollständig verschwunden. Wenn man andererseits die Werte des Eiwesses und des k.o.D. in Blut und Exsudat beobachtet, so sieht man, dass, wenn auch in der ersten Hälfte der Exsudatansammlung ihre Änderungen keine bestimmte Richtung zeigen und man folglich nicht von ihrer Bedeutung sprechen kann, in der zweiten Hälfte, d.h. in der Zeit, in der die Resorption des Exsudates beginnt und das Exsudat endlich vollständig verschwindet, bei den 4 Fällen, bei denen es 12 Tage lang vorhanden ist, der Eiweisspiegel und der k.o.D. des Blutes allmählich sich deutlich steigern, während die entsprechenden Werte des Exsudates nach und nach sinken. Bei einem Falle, bei dem das Exsudat 17 Tage lang yorhanden war, vermehrten sich Bluteiweiss und k.o.D., während der k.o.D. des Exsudates sank, ohne dass zugleich der Eiweissgehalt abnahm. Bei den 2 Fällen, bei denen das Exsudat am längsten vorhanden war, schwankten $\mathrm{Ab}$ - und Zunahme und waren derartige Beziehungen nicht deutlich erkennbar. Kurz, man kann wohl sagen, dass in den Kontrollbestimmungen, wenn die Exsudatresorption verhältnismässig schnell vor sich geht, im Resorptionsstadium das Eiweiss und der k.o.D. des Blutes zunehmen, während die entsprechenden Werte des Exsudates zum Sinken neigen (Fig. 1). Diese. Tatsache wurde von Nishiy a mat and Hayas $i^{11}$ ebenfalls bei Menschenpleuritis beobachtet und auf gleiche Weise wie in der Zusammenfassung von $\mathrm{Nakazawa}^{8)}$. gedeutet. Darum sei sie hier nicht berührt.

Fig. 1. Prozentige Veränderungen des Eiwcisses und dessen kolloid-osmotischen Drucks des Blutes und des Exsudates bei durch, Ameisensäureeinführung hervorgerufener experimenteller Pleuritis.

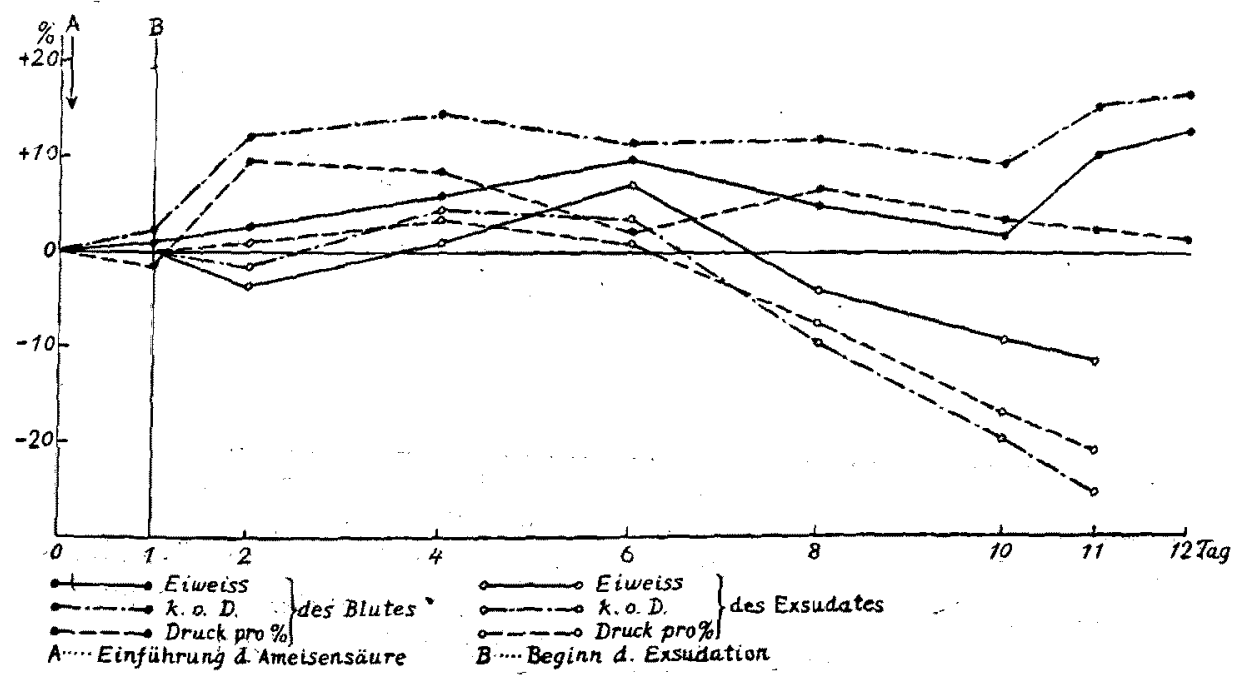




\section{Pyrazolonderivate-Novalgin.}

Nach den Studien einer Reihe von Forschern (Eppinger, Eppinger, Kaunitz u. Popper, ${ }^{10)}$ Winternitz-Korányi, ${ }^{11}$ Hoff u. Schönbauer, ${ }^{12}$ Risak, ${ }^{13)}$ Hitzenberger, ${ }^{14}$ Weitz$m a n n^{15)}$ ) beugt das Pyrazolonderivate-Novalgin infolge seiner gefässdichtenden Wirkung serösen Entzündungen vor und beschleunigt überdies die Heilung derartiger Vorgänge. Unser Kollege $\mathrm{Hayas} \mathrm{i}^{1 /}$ hat dies bei Menschenpleuritis bestätigt. Wie wirkt es nur auf die durch Ameisensäure bei Kaninchen in der Pleurahöhle hervorgerufene Exsudatansammlung?

$\mathrm{Zu}$ diesen Versuchen dienten 4 Kaninchen. Gleichzeitig mit der Ameisensäureeinführung wurde täglich einmal $1 \mathrm{ccm} 5 \%$ Novalgin (Bayer) intravenös dargereicht. Wenn auch die Exsudation fast ähnlich wie in den Kontrollversuchen beginnt, ist die Ansammlung des Exsudates geringer und ist das Exsudat nach Ablauf von 4-5 Tagen schon vollständig verschwunden. Eiweiss und k.o.D. des Blutes und Exsudates verändern sich bei allen $4 \mathrm{Fällen} \mathrm{völlig} \mathrm{gleichartig.} \mathrm{Infolge} \mathrm{der} \mathrm{Novalgin-}$ Wirkung werden nämlich nicht nur Dauer und Grad der Exsudatansammlung herabgesetzt, sondern im Resorptionsstadium werden auch stets Steigerung des Bluteiweisses sowie seines k.o.D. und Herabsetzung des Exsudateiweisses sowie seines k.o.D. beobachtet, ebenso wie es bei den leichten Fällen der Kontrollversuche der Fall war (Fig. 2).

Fig. 2. Prozentige Veränderungen des Eiweisses und dessen kolloid-osmotischen Drucks des Blutes und des Exsudates bei experimenteller Pleuritis unter Einwirkung des Novalgins.

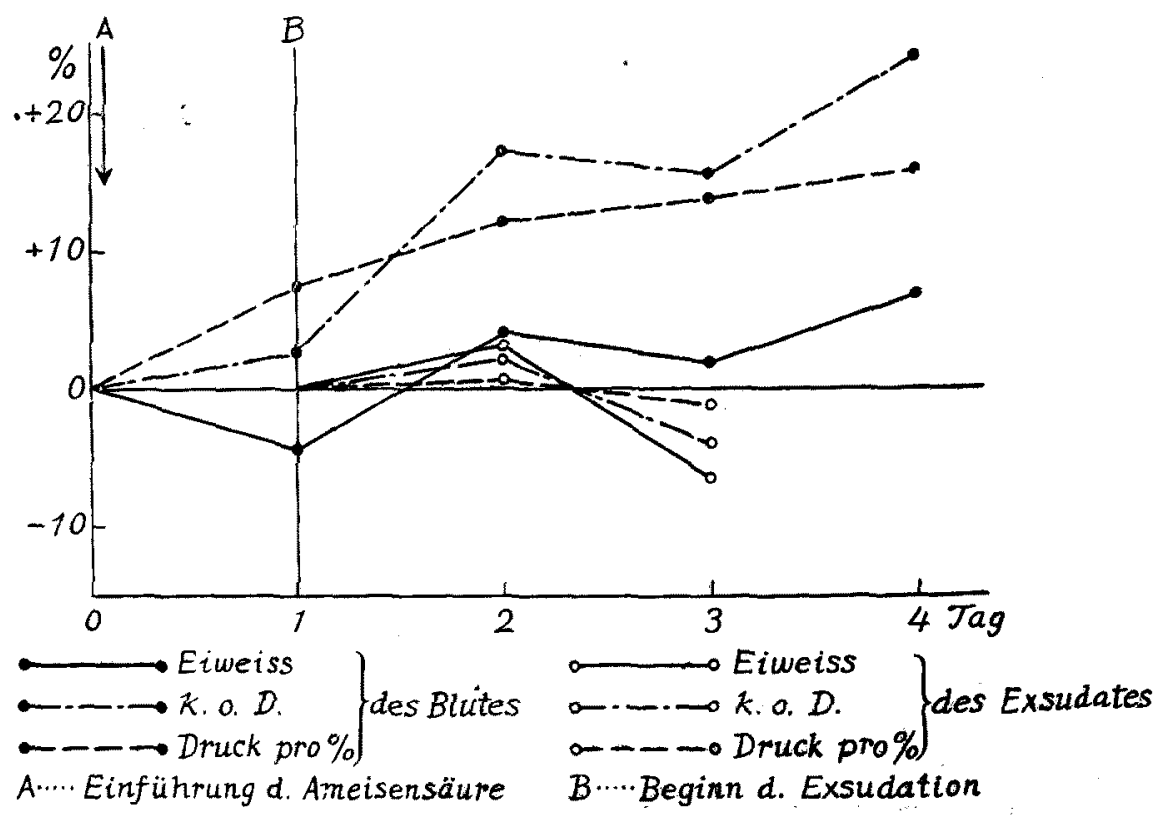


3. Galcium.

Über die Tatsachē, dass das Calcium auf Pleuritis und andere seröse Entzündungen durch Verdichtung der Kapillarwand antiphlogistisch wirkt, liegen zwar schon seit langem Angaben vieler Autoren (Böh m, ${ }^{16)}$ Wright, ${ }^{17}$ Overton, ${ }^{18)}$ Chiani u. Fröhlich, ${ }^{19}$ Chiani u. Januschke, ${ }^{20}$ Starkenstein, ${ }^{211}$ Hamburger, ${ }^{221}$ Mc. Cutcheon u. Lucke,23) Schlück, ${ }^{24)} \mathrm{Kriech}{ }^{25)}$ ) vor. Tierisch-experimentell ist die Frage aber noch nicht ganz geklärt. Darum wurde in folgenden Versuchen untersucht, wie Calcium bei der durch Ameisensäure herbeigeführten experimentellen Pleuritis die Ansammlung und Resorption des Exsudates, sowie das Eiweiss und den k.o.D. in Blut und Exsudat beeinflusst. Als Calcium wurden Takamol (3\% Calcium Salicylicum), $10 \%$ iges $\mathrm{CaCl}_{2}$ und $2 \%$ iges $\mathrm{CaCl}_{2}$ verwendet. Das Takamol und dàs $10 \%$ ige $\mathrm{CaCl}_{2}$ wurden mit der Einführung von Ameisensäure in die Pleurahöhle täglich einmal intravenös dargereicht, das $2 \%$ ige $\mathrm{CaCl}_{2}$ wurde mit Beginn der Exsudation gleichzeitig in die betreffende Pleurahöhle eingeführt, was täglich bis zum Verschwinden des Exsudates wiederholt wurde. Die einmaligen Dosen betrugen $5 \mathrm{ccm}$ bei Takamol, $2 \mathrm{ccm}$ bei $10 \% \mathrm{CaCl}_{2}$ und $2 \mathrm{ccm}$ bei $2 \% \mathrm{CaCl}_{2}$.

Fig. 3. Prozentige Veränderungen des Eiweisses und dessen kolloid-osmotischen Druck des Blutes und des Exsudates bei experimenteller Pleuritis unter Einwirkung des Takamols.

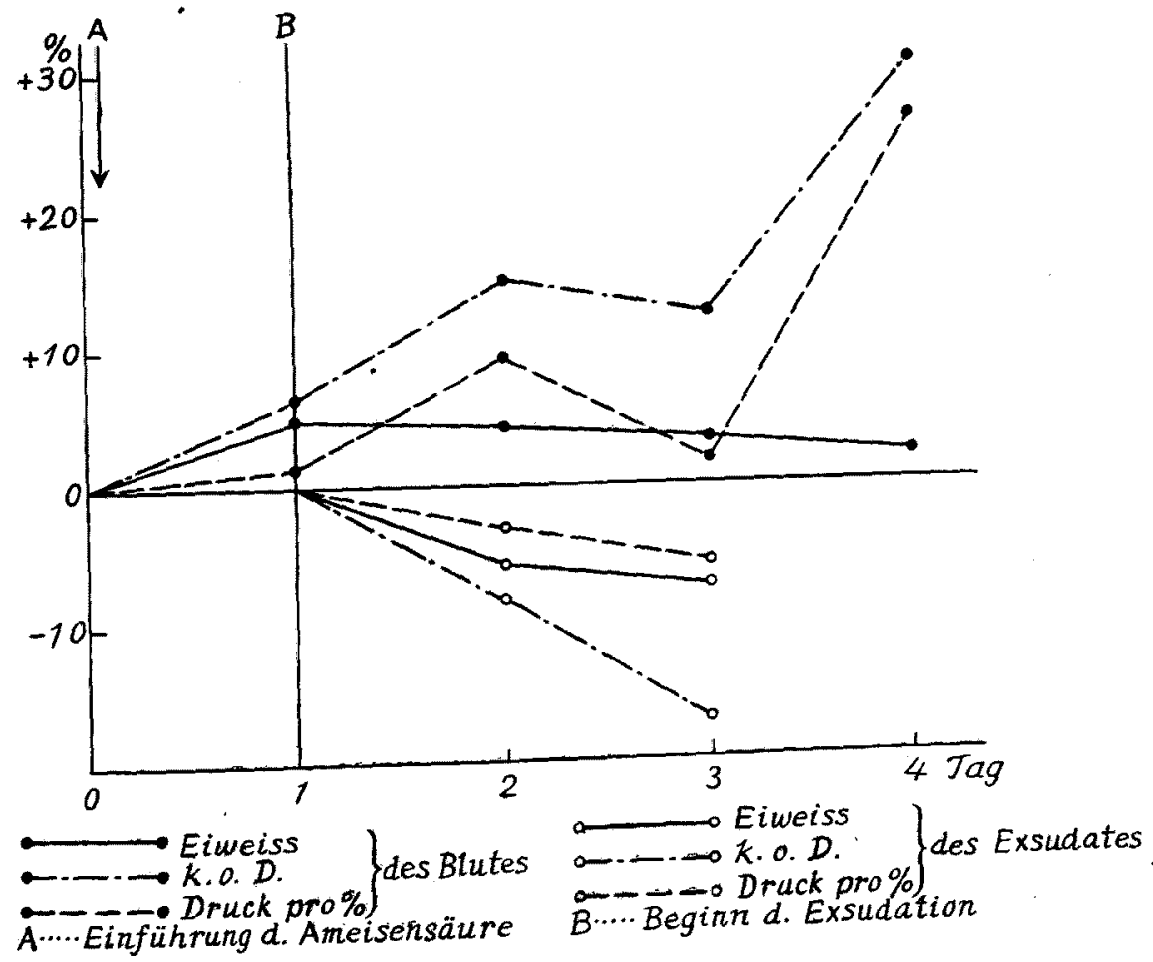


i) Takamol (intravenöse Anwendung): Die Versuche wurden an 5 Kaninchen durchgeführt. Aus den Resultaten ergab sich, dass ohne Ausnahme, wenn auch die Exsudation annähernd gleich wie bëi den Kontrollversuchen beginnt, die Ansammlung des Exsudates geringer ist und nach Ablauf von 3-5. Tagen schon mit dem Beginn der Exsudatresorption das Eiweiss, insbesondere der k.o.D. im.Blut zunimmt, während sie sich im Exsudat verringern (Fig.3).

ii) $10 \% \mathrm{CaCl}_{2}$ (intravenöse Anwendung): Aus den Versuchen an 5 Kaninchen werden im grossen ganzen gleiche Ergebnisse wie in den Takamal-Versuchen erzielt (Fig. 4).

iii) $2 \% \mathrm{CaCl}_{2}$ (intrapleurale Anwendung): Die 5 Versuchstiere zeigen in diesen Versuchen im grossen ganzen gleiche Resultate wie in den beiden oben erwähnten Versuchen.

Aus dem Gesagten geht also hervor, dass die angewendeten Cálcium. präparate sowohl bei intravenöser, als auch bei intrapleuraler Anwendung deutlich die Exsudat-Bildung hemmen und seine Resorption beschleunigen.

Fig. 4. Prozentige Veränderungen des Eiweisses und dessen kolliod-osmotischen Drucks des Blutes und des Exsudates bei experimenteller Pleuritis unter Einwirkung des Calciums (10\% $\mathrm{CaCl}_{2}$ ).

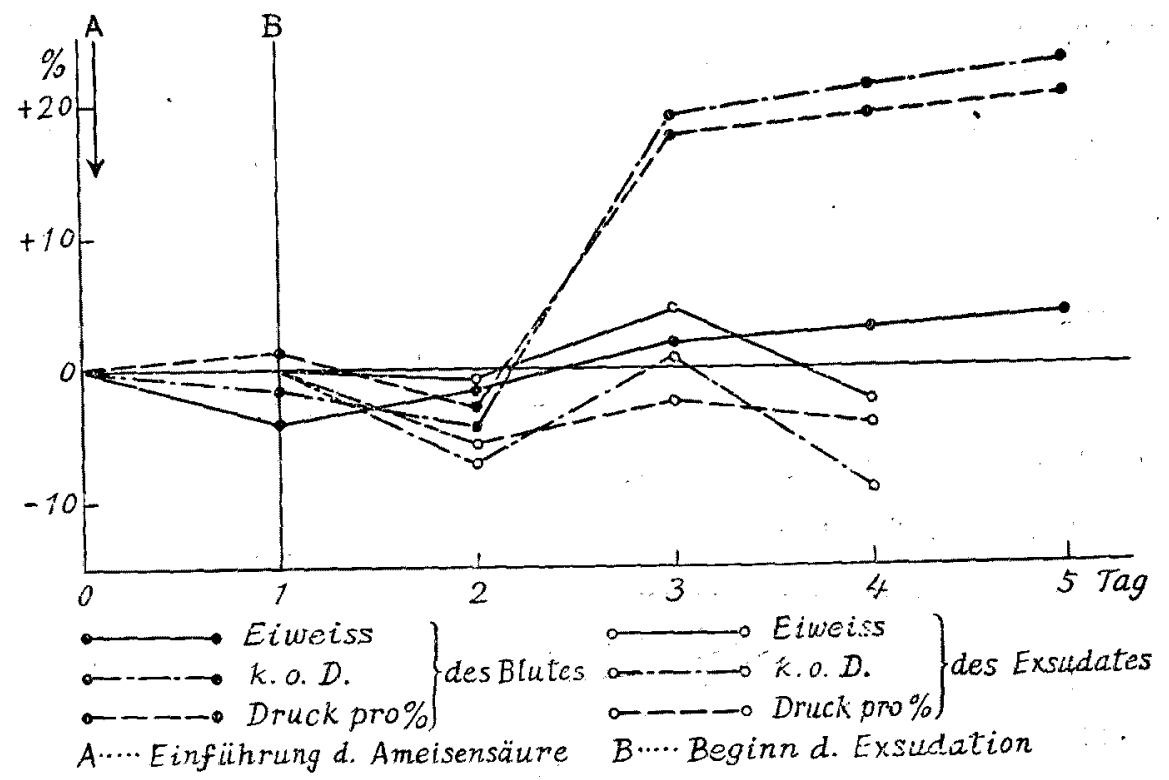

Schluss.

1. Wenn man normalen Kaninchen $1 \mathrm{ccm} 5 \%$ iger Ameisensäure intrapleural einverleibt, so sammelt sich das Exsudat nach 16-18 Stunden in der Pleurahöhle auf der betreffenden Seite an und füllt sie nach 4-5 Tagen völlig. Danach nimmt das Exsudat spontan ab, und bei den 
Fällen, bei denen es nach Ablauf von 12-19 Tagen vollständig verschwunden ist, steigen im Resorptionsstadium, wenn die Resorption verhältnismässig schnell vollendet, Eiweisspiegel und k.o.D. des Blutes allmählich, während sie im Exsudat nach und nach sinken.

2. Auf die oben erwähnte experimentelle Pleuritis wirken Novalgin und Calcium-präparate deutlich hemmend und führen im Resorptionsstadium stets Steigen des Eiweisspiegels und des k.o.D. im Blut und Sinken derselben im Exsudat herbei.

Zum Schluss spreche ich Herrn Prof. Nakazawa für seine freundliche Leitung bei dieser Arbeit meinen herzlichen Dank aus.

\section{Literatur.}

(1) H a y a si, Tohoku Journ. Exp. Med., 1942, 44, 227.

(2) Iversen u. Nakazawa, Biochem. Ztschr., 1927, 191, 307.

(3) Kylin, Acuta med. scandinav., 1929, 72, 561.

(4) Meyer u. Friedheim, Zischr. f. kl. Med., 1931, 119, 236.

(5) Iversen u. Johansen, Klin. Wschr., 1929, 309.

(6) Nishiy a ma, Tohoku Jour. Exp. Med., 1934, 22, 505.

(7) Krogh u. Nakazawa, Biochem. Ztschr., 1927, 188, 241.

(8) Nakazawa, Nippon Naikagakkai Zassi, 1943, 31, 311.

(9) Eppinger, Wien. klin. Wschr., 1935, 1313.

(10) Eppinger, Kaunitz u. Popper, Die seröse Entzündung, Wien 1935, 245.

(11) Winternitz-Korányi, Dtsch. med. Wschr., 1930, 1779.

(12) Hoff u. Schönbauer, Ibid., 1935, 786.

(13) Risak, Ztschr. f. klin. Med., 1935, 128, 583.

(14) Hitzenberger, Med. klin., 1935, 1331.

(15) Weitsmann, Ztschr.f. klin. Med., 1940, 137, 429.

(16) Bö hm, Arch.f. exp. Path. u. Pharm., 1876, 5, 329.

(17) Wright, Lancet, 1896, 150, 153.

(18) Overton, Arch. f. ges. Physiol., 1904, 105, 279.

(19) Chiari u. Fröhlich, Arch. f. exp. Path. u. Pharm., 1911, 64, 214.

(20) Chiari u. Januschke, Ibid., 1911, 65, 120.

(21) Starkenstein, Münch. med. Wschr., 1919, 205.

(22) Hamburger, Biochem. Zischr., 1922, 129, 153.

(23) Mc. Cutcheon u. Lucke, J. gen. Physipl. 1928, 12, 129.

(24) Schlück, Med. Welt, 1930, 1165.

(25)' Kriech, Z. Tbc., 1935, 72, 31. 